
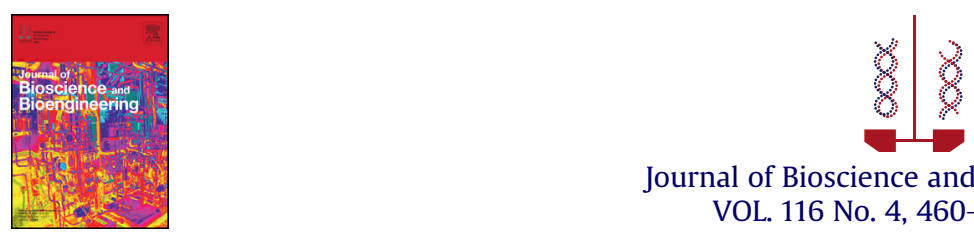

Journal of Bioscience and Bioengineering

VOL. 116 No. 4, 460-464, 2013

\title{
Evaluation of Clostridium ljungdahlii DSM 13528 reference genes in gene expression studies by qRT-PCR
}

\author{
Juanjuan Liu, ${ }^{1,2, \dagger}$ Yang Tan, ${ }^{2, \dagger}$ Xiaohong Yang, ${ }^{1, \dagger}$ Xiaohua Chen, ${ }^{2}$ and Fuli $\mathrm{Li}^{2, *}$
}

Key Laboratory of Horticulture Science for Southern Mountainous Regions, College of Horticulture and Landscape Architecture, Southwest University, Chongqing 400716, China ${ }^{1}$ and Key Laboratory of Biofuels, Qingdao Institute of Bioenergy and Bioprocess Technology, Chinese Academy of Sciences, Qingdao 266101, China ${ }^{2}$

Received 21 December 2012; accepted 8 April 2013

Available online 4 May 2013

\begin{abstract}
Clostridium ljungdahlii DSM 13528 is a promising platform organism for biofuel production from syngas. Gene expression analysis permits a better understanding of the important molecular biological characteristics of this organism, such as carbon fixation and solvent adaptation. Normalization is a prerequisite for accurate gene expression analysis, but until now, no valid reference genes have been proposed for quantitative real-time polymerase chain reaction (qRT-PCR) analysis of C. ljungdahlii DSM 13528. In this study, seven candidate reference genes (gyrA, rho, fotl, rpoA, gukl, recA, 16S rRNA) were selected for qRT-PCR quantification of their expression levels in various culture conditions that corresponded to different carbon sources and stresses. Two analytical programs, geNorm and NormFinder, were used to evaluate reference gene stability. The results showed that gyrA, rho and fotl exhibited the most stable expression levels across all tested samples and can be confidently used as reference genes to normalize the transcriptional data of target genes in qRT-PCR analyses of $C$. ljungdahlii DSM 13528. This study presents the first attempt to explore the validity of candidate reference genes and provide a set of valid reference genes for normalizing C. ljungdahlii DSM 13528 target gene expression and transcriptome analysis.
\end{abstract}

(c) 2013, The Society for Biotechnology, Japan. All rights reserved.

[Key words: Clostridium ljungdahlii; Quantitative real-time PCR; Reference genes; Carbon source; Stress]

Quantitative real-time PCR (qRT-PCR) is a popular molecular technique for gene expression studies (1). This technique has the advantages of good reproducibility, high sensitivity and specificity (2-4). qRT-PCR has been used in a number of biological fields, including pathogen detection and polymorphism analyses of single nucleic acids (5-8). However, in analyses of gene expression levels, differences in the amounts of starting materials, the RNA quality and the cDNA sample loading might decrease qRTPCR accuracy. Therefore, it is necessary to select one or more appropriate reference genes with which to normalize qRT-PCR data.

In recent years, reference gene evaluations were reported in different bacteria, including Staphylococcus aureus (9), Clostridium difficile (10), Bacillus cereus (11), Gluconacetobacter diazotrophicus (12) and Oenococcus oeni (13). However, the results of these studies were inconsistent, and an ideal universal reference gene has not been proposed. Thus, reference genes should be validated prior to experiments.

Because traditional fuels, such as petroleum, coal and gas, are limited and environmental consequences have become increasingly severe due to the burning of these fuels, there is an urgent need for the development of sustainable and environmentally friendly fuels. One alternative, bioenergy, has received increasing

\footnotetext{
* Corresponding author. Tel.: +86 532 80662655; fax: +86 53280662778.

E-mail addresses: lifl@qibebt.ac.cn, lifuli@gmail.com (F. Li).

The first three authors contributed equally to this work.
}

amounts of attention. Biomass-derived biofuels have shown great potential (14). Technologies that ferment syngas into biofuels are considered to be most attractive because they have the advantage of utilizing whole biomass $(15,16)$. The syngas-utilizing bacteria Clostridium ljungdahlii has attracted a wider range of attention due to its high yields of ethanol and high growth rate. Furthermore, the publication of the C. ljungdahlii DSM 13528 genome and the establishment of a genetic transformation system for this organism have made it a model organism for studies of syngas fermentation into biofuels.

C. ljungdahlii DSM 13528 fixes $\mathrm{CO}$ and $\mathrm{CO}_{2}$ through the WoodLjungdahl pathway and converts syngas into acetyl-CoA, a precursor for the production of many useful chemicals, such as ethanol (17), acetate (18), butanol (19), 2,3-butanediol (20) and other byproducts. The solvents generated by C. ljungdahlii DSM 13528 are autotoxic, and thus, the strain has evolved mechanisms to acclimate to these stresses $(19,20)$. The publication of the C. ljungdahlii DSM 13528 genome has permitted the identification of the genes involved in carbon fixation, sugar metabolism, solvent synthesis, quorum sensing and two-component signal system, among others. Additionally, some alcohol dehydrogenases and acetaldehyde dehydrogenases that are possibly involved in ethanol and butanol consumption have also been identified (19). Moreover, the genome sequence allowed us to analyze gene expression for a better understanding of the general physiology of the bacteria and, in particular, to decipher the carbon fixation and solvent-adapting pathways. 
Gene expression analyses with qRT-PCR can provide useful information for the elucidating of physiological processes at the molecular level. However, until now, no ideal reference genes had been proposed for gene expression analyses of C. ljungdahlii DSM 13528. Köpke et al. (20) used the formate tetrahydrofolate ligase and guanylate kinase genes as references in studies of the expression profiles of genes related to 2,3-butanediol production in the acetogenic bacterium Clostridium autoethanogenum. However, that study did not assess the validity of the two reference genes in other acetogenic bacteria. In this study, we used qRT-PCR to analyze the expression patterns of seven C. ljungdahlii DSM 13528 candidate genes in five tested conditions. Furthermore, we comprehensively evaluated the validity of these seven genes with geNorm v3.5 and NormFinder and provided a useful set of valid reference genes for gene expression analyses in C. ljungdahlii DSM 13528.

\section{MATERIALS AND METHODS}

Strain and culture conditions $\quad$ C. ljungdahlii DSM 13528 was purchased from a German collection of microorganisms and cell cultures (DSMZ, Braunschweig, Germany) and was grown anaerobically under the following five tested conditions in PETC medium (17) at $37^{\circ} \mathrm{C}$ (in triplicate): (i) $5 \mathrm{~g} / \mathrm{l}$ of fructose as the sole carbon source, (ii) syngas ( $\mathrm{CO}: \mathrm{CO}_{2}, 4: 1,1.6 \mathrm{~atm}$ ) as the sole carbon source, (iii) $5 \mathrm{~g} / \mathrm{l}$ of fructose supplemented with $0.5 \%$ ethanol, (iv) $5 \mathrm{~g} / \mathrm{l}$ of fructose supplemented with $2 \% 2$, 3-butanediol, and (v) $5 \mathrm{~g} / \mathrm{l}$ of fructose supplemented with $0.25 \%$ butanol.

The growth profiles under each experimental condition were monitored by measuring the optical density at $600 \mathrm{~nm}\left(\mathrm{OD}_{600 \mathrm{~nm}}\right)$ with a visible spectrophotometer (Unico, Shanghai, China). Cells in the different media were sampled during the early exponential growth, middle exponential and stationary phases. A total of 45 samples were immediately frozen in liquid nitrogen and stored at $-80^{\circ} \mathrm{C}$ before RNA extraction.

Nucleic acid extraction and cDNA synthesis Genomic DNA was extracted as previously described (20). Total RNA was isolated with the RNA mini kit (Qiagen, Hilden, Germany) according to the manufacturer's instructions. Next, the total RNA sample was treated with DNase, and the elimination of genomic DNA was determined by quantitative RT-PCR. The concentration and purity of the RNA samples were determined with a NanoVue spectrophotometer (GE Healthcare Bioscience Corp., Piscataway, NJ, USA). The $\mathrm{OD}_{260 \mathrm{~nm}} / \mathrm{OD}_{280 \mathrm{~nm}}$ and $\mathrm{OD}_{260 \mathrm{~nm}} / \mathrm{OD}_{230 \mathrm{~nm}}$ were determined to evaluate the purity of the RNA samples, and the RNA sample integrity was verified by $1 \%$ agarose gel electrophoresis. Reverse transcription reactions ( $1 \mu \mathrm{g}$ of total RNAs) were performed with the QuantiTect Reverse Transcription Kit (Qiagen) according to the manufacturer's instructions.

Primer design and determination of PCR efficiency A total of ten pairs of specific primers for reference genes and target genes were designed with the Primer 5 open software package (Premier Biosoft, Palo Alto, CA, USA), and the details of these primers are provided in Supplementary Table S1. The DNA fragments that corresponded to the candidate reference genes were amplified, purified and cloned into the vector pMD-18T (Takara, Dalian, China) to generate a standard curve with which to calculate the PCR efficiency. The constructed plasmids were transformed into the competent Escherichia coli DH5 $\alpha$. The cells were plated on LB medium with $100 \mu \mathrm{g} / \mathrm{ml}$ ampicillin, and the colonies were verified by PCR. The identity of the insert fragments was confirmed by sequencing. Plasmid DNA was extracted with a plasmid kit (Qiagen) and the concentrations were determined with NanoVue spectrophotometer. A 10 -fold dilution series was prepared in triplicate for qRT-PCR analysis. The qRT-PCR reactions were performed in a total volume of $20 \mu \mathrm{l}$ that contained $10 \mu \mathrm{l}$ of $2 \times$ SYBR Green mix (Roche, Penzberg, Germany), $0.6 \mu \mathrm{l}$ of each primer, $3 \mu \mathrm{l}$ of template and $5.8 \mu \mathrm{l}$ of sterile distilled water. The reaction mixtures were incubated for $10 \mathrm{~min}$ at $95^{\circ} \mathrm{C}$, followed by 40 amplification cycles of $15 \mathrm{~s}$ at $95^{\circ} \mathrm{C}$ and $60 \mathrm{~s}$ at $60^{\circ} \mathrm{C}$. All reactions were performed in 96-well reaction plates. A log 10 series of triplicate dilutions of reference gene fragment-containing plasmids were investigated, and the PCR efficiencies were determined from the slope of the curve and the formula $\mathrm{E}(100 \%)=\left(10^{(-1 /}\right.$ slope) -1$)^{*} 100 \%$. The correlation coefficients were also calculated from the curves. A dissociation curve was also generated for every pair of primers to investigate the specificity of the primers. The intra-assay CVs were evaluated according to a previously described method (21).

Reference gene validation and target gene normalization The expression levels of all candidate reference genes were detected by qRT-PCR in each cDNA sample. qRT-PCR was performed as described above. The candidate reference gene stability was analyzed using geNorm v3.5 (1) and NormFinder (22). To evaluate the reference genes, the same cDNA samples from the carbon source group during the early exponential growth phase were subjected to qRT-PCR to analyze the expression profiles of three target genes (csta, mthfr and aor); the details of these target genes are shown in Supplementary Table S1. The relative quantification (RQ) for every target gene was calculated based on the $2^{-\Delta \Delta C t}$ method.
TABLE 1. Candidate reference genes and target genes from Clostridium ljungdahlii DSM 13528.

\begin{tabular}{|c|c|c|}
\hline $\begin{array}{l}\text { Gene } \\
\text { symbol }\end{array}$ & Function & Pathways \\
\hline fotl & Formate tetrahydrofolate ligase & CO fixation \\
\hline $16 S$ rRNA & 16S ribosomal RNA subunit & Translation \\
\hline recA & Recombinase A & $\begin{array}{l}\text { Homologous } \\
\text { recombination }\end{array}$ \\
\hline gukl & Guanylate kinase & Nucleotide metabolism \\
\hline gyrA & Gryrase subunit A & DNA replication \\
\hline rho & Transcriptional termination factor & Transcription \\
\hline rpoA & $\begin{array}{l}\text { DNA-directed RNA polymerase } \\
\text { subunit } \alpha\end{array}$ & Transcription \\
\hline csta & Carbon starvation protein $\mathrm{A}$ & Carbon global regulation \\
\hline mthfr & Methylenetetrahydrofolate reductase & $\mathrm{CH} 3-\mathrm{THF}$ formation \\
\hline aor & $\begin{array}{l}\text { Tungsten-containing aldehyde } \\
\text { ferredoxin oxidoreductase }\end{array}$ & Ethanol production \\
\hline
\end{tabular}

\section{RESULTS}

Choice of candidate reference genes and primer validation To minimize the risk of co-regulation, seven candidate reference genes that functioned in different pathways were selected (Table 1). To evaluate the specificity and amplification efficiency of each pair of primers, 10 -fold serial dilutions of plasmid DNA (each plasmid carried the target fragment of every candidate gene) were used for qPCR amplification. The generated standard curve showed that the amplification efficiency of the primers ranged from $96 \%$ to $103 \%$. The amplification of each gene was performed under the same thermal cycle conditions, and all primers produced a single melting curve peak (data not shown). The correlation coefficients were determined from the resulting standard curves and ranged from 0.994 to 1.000 . The intra-assay CVs ranged from $1.41 \%$ to $6.67 \%$, indicating the precision and robustness of the assay.

Expression levels of the candidate reference genes RNA expression levels of the seven candidate reference genes were measured in triplicate under the five tested conditions during three growth phases. Initially, descriptive statistics of the expression levels in all 45 samples were calculated for each gene (Table 2 ). The mean $\mathrm{Ct}$ values ranged from 16.77 to 28.80. The highest expression levels were observed with the candidate reference genes $16 \mathrm{~S}$ rRNA and fotl, with mean Ct values of 16.77 and 20.50, respectively. The candidate reference genes rho and gukl exhibited the lowest expression levels, with mean Ct values of 28.80 and 27.94, respectively. The standard deviation (SD) was also calculated for each gene. The results showed that the expression levels of $r p o A$ $(\mathrm{SD}=1.17)$ and $\operatorname{rec} A(\mathrm{SD}=1.40)$ had the most variation, which indicated the low expression stability of these two genes. In comparison, the SD values for rho (SD = 0.73) and gyrA $(S D=0.80)$ were the lowest across all of the samples, which suggested high expression stability of these two genes.

Stability of reference gene expression Two analytical programs, geNorm (1) and NormFinder (22), were used to evaluate the expression stability of the seven candidate reference genes and determine the optimal choice and number of the reference genes. GeNorm is a useful tool for the selection of the most stable internal control genes according to the principle that the expression ratio of two ideal internal control genes will be identical in all tested samples. In analyses with this software, the $M$ value is the average pairwise variation of a particular gene with all other control genes. A lower $M$ value represents more stable expression. If the $M$ value is below the cut-off value of 1.5 , the gene has a stable expression level. Additionally, NormFinder uses another algorithm to identify the optimal normalization gene among a set of candidate reference genes by ranking the various candidate reference genes according to their inter- and 
TABLE 2. Descriptive statistics of candidate reference genes.

\begin{tabular}{|c|c|c|c|c|c|c|c|}
\hline \multirow[t]{2}{*}{ Parameter } & \multicolumn{7}{|c|}{ Genes } \\
\hline & gyrA & rho & гроA & 16S rRNA & gukl & fotl & recA \\
\hline $\mathrm{GM}[\mathrm{Ct}]$ & 27.67 & 28.80 & 26.80 & 16.77 & 27.94 & 20.50 & 24.39 \\
\hline $\mathrm{n}[\mathrm{mRNA}]$ & $6.95 \times 10^{4}$ & $1.95 \times 10^{4}$ & $2.14 \times 10^{4}$ & $1.54 \times 10^{5}$ & $2.82 \times 10^{4}$ & $1.59 \times 10^{5}$ & $1.0 \times 10^{5}$ \\
\hline $\mathrm{AM}[\mathrm{Ct}]$ & 27.69 & 28.81 & 26.83 & 16.83 & 27.97 & 20.52 & 24.45 \\
\hline $\operatorname{Min}[C t]$ & 26.07 & 27.12 & 24.83 & 14.74 & 26.14 & 18.58 & 21.60 \\
\hline $\operatorname{Max}[\mathrm{Ct}]$ & 29.88 & 31.18 & 29.27 & 20.09 & 30.97 & 22.61 & 28.14 \\
\hline $\mathrm{SD}[ \pm \mathrm{Ct}]$ & 0.80 & 0.73 & 1.17 & 1.15 & 0.99 & 0.83 & 1.40 \\
\hline $\mathrm{CV}[\% \mathrm{Ct}]$ & 2.90 & 2.53 & 4.38 & 6.81 & 3.54 & 4.06 & 5.72 \\
\hline
\end{tabular}

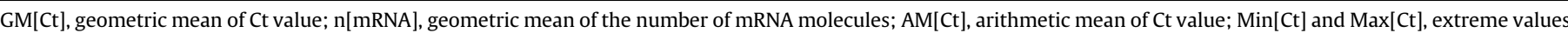
of $\mathrm{Ct}$; $\mathrm{SD}[ \pm \mathrm{Ct}]$, standard deviation of $\mathrm{Ct}$ value and the number of mRNA molecules; $\mathrm{CV}[\% \mathrm{Ct}]$, coefficient of variance expressed as percentage of $\mathrm{Ct}$ value.

intragroup expression variation (S value). The most stable reference gene will have the lowest $S$ value.

Fig. 1A shows the expression stability of the investigated candidate reference genes across the 45 samples as calculated by geNorm. According to the geNorm analysis, genes rho $(M=0.783)$, gyrA $(M=0.798)$ and fotl $(M=0.864)$ had the lowest average $M$ value, which indicated the high expression stability of these three genes. The genes recA $(M=1.516)$ and $16 S \operatorname{rRNA}(M=1.572)$ had the highest $M$ values among the seven candidate genes, both of which exceeded the cut-off value of 1.5 . Thus, it was suggested that rec $A$ and $16 S$ rRNA should be considered the least stable genes across the set conditions. Additionally, NormFinder was used to analyze the expression stability of the candidate genes (Table 3). The NormFinder analysis provided results similar to those of the geNorm analysis. Of the seven candidate genes, rho $(S=0.089)$, gyrA $(S=0.109)$ and fotl $(S=0.196)$ had the most stable expression levels across all of the samples. In contrast, $\operatorname{rec} A(S=0.962)$ and $16 S$ rRNA $(S=1.007)$ had the least stable expression levels.

Furthermore, the stability of the seven candidate genes in two distinct series of treatments (carbon source and stress) was also evaluated using geNorm (Supplementary Fig. S1 and Fig. 2A) and NormFinder (Table 3 and Fig. 2B). The distributions of stability for

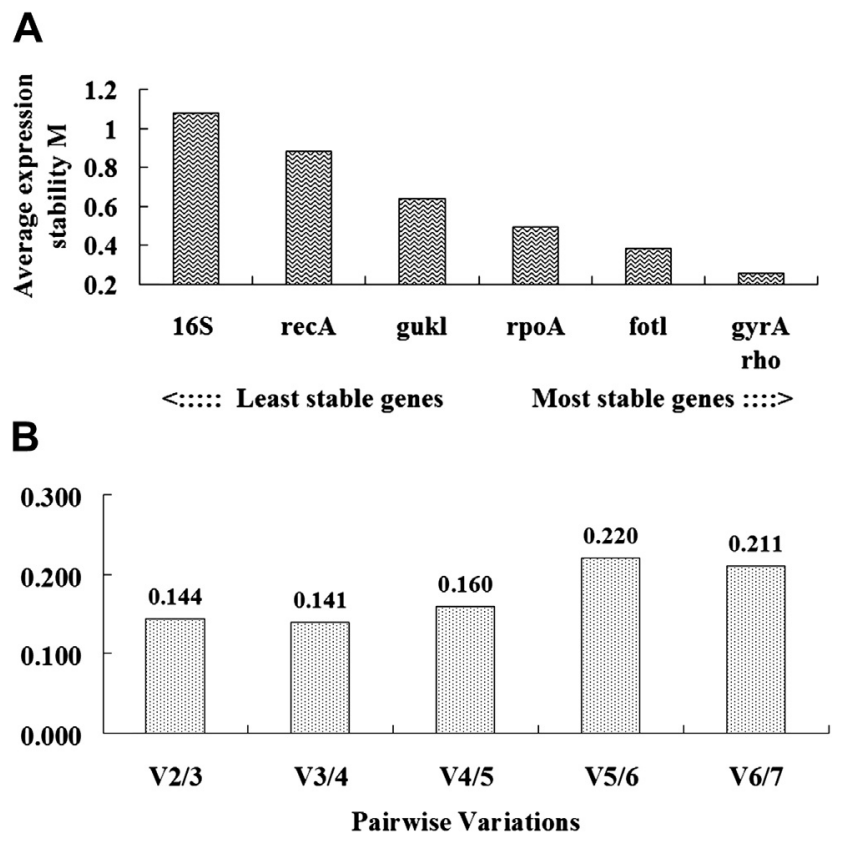

FIG. 1. geNorm analysis of reference genes in Clostridium ljungdahlii DSM 13528. (A) The average expression stability $(M)$ of all candidate reference genes. On the horizontal axis, the more stably expressed genes are positioned on the right side of the diagram, and the less stably expressed genes are on the left side. (B) Determination of the optimal number of control genes required for reliable normalization. these seven candidate genes in response to carbon source or stress treatments were similar to those of all of the samples. In the cells subjected to the carbon source and stress treatments, rho, gyrA and fotl were most stably expressed and both recA and $16 S$ rRNA were least stably expressed. In conclusion, the genes rho, gyrA and fotl are recommended as applicable reference genes for the normalization of target gene expression.

Determination of the optimal number of reference genes To determine the optimal number of reference genes, a pairwise variation $\left(V_{\mathrm{n}} / V_{\mathrm{n}+1}\right)$ was calculated by geNorm. According to Vandesompele's conclusion, 0.15 was usually used as the cut-off value with which to determine the optimal number of reference genes (1) such that if $V_{\mathrm{n}} / V_{\mathrm{n}+1}<0.15$, the inclusion of an additional reference gene is not required. All samples were included in the calculations of the optimal number of reference genes by geNorm (Fig. 1B). In this study, the value of $V_{\mathrm{n}} / V_{\mathrm{n}+1}$ ranged from 0.141 for $V_{2 / 3}$ to 0.211 for $V_{6 / 7}$. The value for $V_{2} / V_{3}(0.144)$ was below the cut-off of 0.15 , which implied that the genes gyrA and rho were sufficient and likely to normalize the expression of target genes. Additionally, geNorm also indicated the optimal number of reference genes for the following distinct series of treatments. In the carbon source group (Supplementary Fig. S1B), the $V_{2} / V_{3}$ value (0.094) was much lower than the cut-off value, indicating that the reference genes gyrA and rho were sufficient. However, the reference genes gyrA, rho and fotl were sufficient to generate a confident result in the stress group (Supplementary Fig. S1D), as the $V_{3} / V_{4}$ value was 0.137 .

Use of the validated factor to normalize the expression of a target gene The validated factors were used to normalize three target genes to evaluate the ability of this method to detect changes in transcript abundance. Two genes involved in carbon utilization (cata and mthfr) and one gene involved in the ethanol synthetic pathway (aor) were analyzed. The expression levels of the three genes were predicted to be higher in the presence of syngas than in the presence of fructose during the early exponential growth phase. The combination of rho and gyrA was used to normalize the expression of the three genes. The transcriptional abundances of csta, mthfr and aor were 2-3-fold higher in the presence of syngas than in the presence of fructose (Fig. 3). Thus, the reference genes

TABLE 3. Expression stability values (S) of the seven candidate reference genes in Clostridium ljungdahlii DSM 13528.

\begin{tabular}{|c|c|c|c|c|c|c|}
\hline \multirow[t]{2}{*}{ Rank } & \multicolumn{2}{|c|}{ Total } & \multicolumn{2}{|c|}{ Carbon source } & \multicolumn{2}{|c|}{ Stress } \\
\hline & Gene & Stability & Gene & Stability & Gene & Stability \\
\hline 1 & rho & 0.089 & gyrA & 0.085 & gyrA & 0.083 \\
\hline 2 & gyrA & 0.109 & rho & 0.085 & rho & 0.083 \\
\hline 3 & fotl & 0.196 & fotl & 0.100 & rроA & 0.336 \\
\hline 4 & rpoA & 0.429 & gukl & 0.310 & fotl & 0.353 \\
\hline 5 & gukl & 0.480 & гроА & 0.520 & recA & 0.435 \\
\hline 6 & recA & 0.962 & recA & 0.797 & gukl & 0.497 \\
\hline 7 & $16 S$ rRNA & 1.007 & $16 S$ rRNA & 1.200 & $16 S$ rRNA & 0.674 \\
\hline
\end{tabular}


A

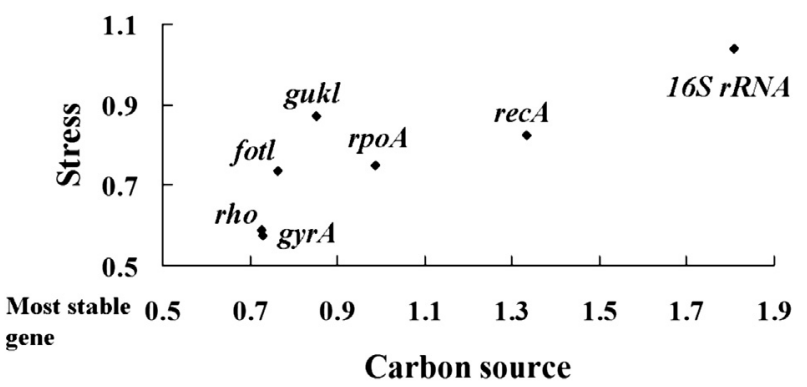

B

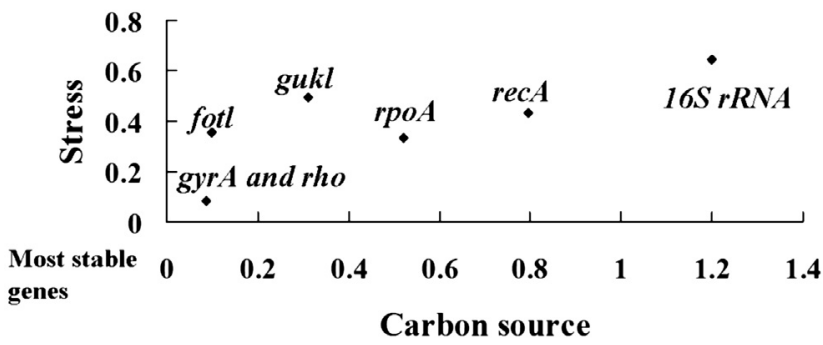

FIG. 2. Ranking of the stability of the reference genes between the two treatment groups, including different carbon sources and different stress treatments. Genes were ordered by both geNorm (A) and NormFinder (B) and were plotted on two axes corresponding to the two types of tested culture conditions. The horizontal axes in $A$ and $B$ represent the $M$ and $S$ values in carbon source treatments for each gene, respectively. The vertical axes in A and B represent the $M$ and $S$ values in stress treatments for each gene, respectively. The position of each gene on the 2-D graph indicates its stability as a reference gene and thus the most stable gene was located at the left bottom position.

validated in this study were appropriate for the detection of changes in gene expression.

\section{DISCUSSION}

Currently, qRT-PCR is one of the most widely used methods of gene expression analysis in a number of biological systems, such as bacteria $(9,23)$ and plants $(24,25)$. It is essential to select stable reference genes to ensure the accuracy of this technology. An ideal reference gene should have a constant expression level, regardless of the experimental conditions. However, until now, the expression of any single gene had not been verified to remain constant under any experimental condition. The selection of invalidated reference genes with which to normalize target gene expression will lead to incorrect interpretations of qRT-PCR results.

qRT-PCR has been performed to study of the expression levels of genes involved in 2,3-butanediol production in one acetogenic

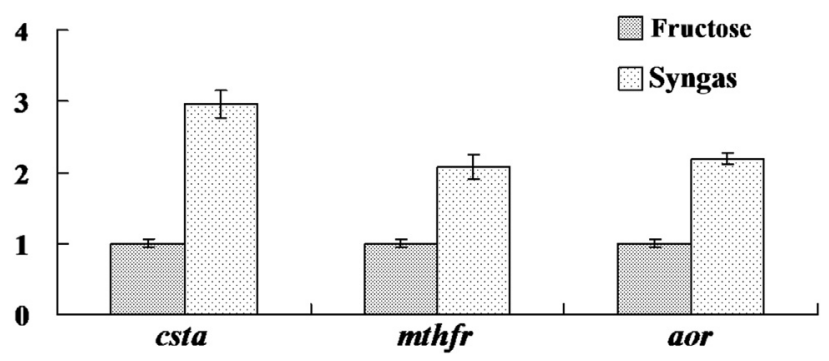

FIG. 3. Relative expression of target genes in Clostridium ljungdahlii DSM 13528 that were cultivated in medium with either fructose or syngas as the sole carbon source, respectively. The rho and gyrA genes were used as reference genes for data normalization. Values are shown as the averages of at least 3 independent determinations; the error bars represent the standard deviation. bacterium; in this study, gukl and fotl were used to normalize the transcriptional abundance of the target genes (20). However, neither of these genes had been previously validated in other acetogenic bacteria. In this study, the expression stability of seven genes under five different conditions during a full life cycle was evaluated for qRT-PCR gene expression analyses of C. ljungdahlii DSM 13528. The combination of gyrA, rho and fotl was recommended as a set of valid internal control genes. The stability of the fotl gene was ranked in the top three genes across all of the tested samples. Acetogens fix inorganic carbon ( $\mathrm{CO}$ and $\mathrm{CO}_{2}$ ) for survival through the Wood-Ljungdahl pathway, in which formate tertrahydrofolate ligase is an essential enzyme. Although fotl is not widely used as a reference gene for normalization, it is considered to be a putative housekeeping gene in acetogens $(26,27)$. Additionally, in this study, the expression of fotl under all five conditions was relatively stable in C. ljungdahlii DSM 13528.

In contrast, the expression levels of gukl varied considerably across all of the investigated samples, and thus, gukl was not recommended as a valid reference gene. The discrepancy might be partly due to the differences in the experimental conditions and species between the two studies. Indeed, the expression levels of the reference genes may vary substantially in response to differences in the experimental conditions and species $(10,28,29)$. Until now, there has been no consensus regarding which gene is appropriate in all qRT-PCR gene expression studies in bacteria. Therefore, validation of the qRT-PCR reference genes is required prior to experiments.

The 16S rRNA gene is widely used as a reference gene in qRT-PCR expression studies of Clostridium (30) and other genus (31,32). However, the transcription level of $16 S$ rRNA was reported to depend on the physiological state of the bacteria (33). Although rRNA accounts for 95\% of total RNA, it is not always representative of the mRNA fraction. The transcription levels of rRNA were previously shown to be 1000 -fold higher than that of mRNA (13). Additionally, the initial analysis of $16 S$ rRNA Ct values indicated that it was expressed at a very high level. The expression levels of most of the target genes were not very high in comparison to $16 S$ rRNA, and therefore, care should be taken when using 16S rRNA as a reference gene $(10,34)$. In this study, $16 S$ rRNA was shown to have a low stable expression level according to the high $M(1.572)$ and S values (1.007), as determined by geNorm and NormFinder, respectively.

Many reports have shown that experimental conditions could significantly affect the expression stability of reference genes $(35,36)$. In this study, the expression stabilities of seven candidate genes in the carbon source and stress groups were ranked differently by geNorm or NormFinder. For example, the geNorm analysis showed that rho $(M=0.728)$ and gyrA $(M=0.729)$ were ranked in the first and second positions in the carbon source group, respectively. In contrast, the opposite trend was observed in the stress group. Additionally, the expression stability of $\operatorname{gukl}(M=0.852)$ was ranked in the fourth position in the carbon source group and the sixth position in the stress group. As far as the discrepancy is concerned, it is extremely important to validate the candidate reference genes to avoid erroneous expression analyses of the target genes by qRT-PCR.

Among the seven candidate reference genes, gyrA, fotl and rho were identified and validated as the most stably expressed. Furthermore, a pairwise analysis indicated that three genes were sufficient to calculate a valid normalization factor. The combination of gyrA, fotl and rho could be used to normalize the expression of target genes and was suitable to detect changes in transcript abundance.

Supplementary data related to this article can be found at http:// dx.doi.org/10.1016/j.jbiosc.2013.04.011 


\section{ACKNOWLEDGMENTS}

This study was supported by the Natural Science Foundation of China (30974409, 31111120078) and the National Basic Research Program of China (2011CB707404).

\section{References}

1. Vandesompele, J., De Preter, K., Pattyn, F., Poppe, B., Van Roy, N., De Paepe, A., and Speleman, F.: Accurate normalization of real-time quantitative RT-PCR data by geometric averaging of multiple internal control genes, Genome Biol., 3, RESEARCH0034 (2002).

2. Garson, J. A., Grant, P. R., Ayliffe, U., Ferns, R. B., and Tedder, R. S.: Real-time PCR quantitation of hepatitis B virus DNA using automated sample preparation and murine cytomegalovirus internal control, J. Virol. Methods, 126, 207-213(2005).

3. Ginzinger, D. G.: Gene quantification using real-time quantitative PCR: an emerging technology hits the mainstream, Exp. Hematol., 30, 503-512 (2002).

4. Held, C. A., Stevens, J., Livak, K. J., and Williams, P. M.: Real time quantitative PCR, Genome Res., 6, 986-994 (1996).

5. Bustin, S. A., Benes, V., Nolan, T., and Pfaffl, M. W.: Quantitative real-time RTPCR-a perspective, J. Mol. Endocrinol., 34, 597-601 (2005).

6. Giulietti, A., Overbergh, L., Valckx, D., Decallonne, B., Bouillon, R., and Mathieu, C.: An overview of real-time quantitative PCR: applications to quantify cytokine gene expression, Methods, 25, 386-401 (2001).

7. Weis, J. H., Tan, S. S., Martin, B. K., and Wittwer, C. T.: Detection of rare mRNAs via quantitative RT-PCR, Trends Genet., 8, 263-264 (1992).

8. Gachon, C., Mingam, A., and Charrier, B.: Real-time PCR: what relevance to plant studies? J. Exp. Bot., 55, 1445-1454 (2004).

9. Theis, T., Skurray, R. A., and Brown, M. H.: Identification of suitable internal controls to study expression of a Staphylococcus aureus multidrug resistance system by quantitative real-time PCR, J. Microbiol. Methods, 70, 355-362 (2007).

10. Metcalf, D., Sharif, S., and Weese, J. S.: Evaluation of candidate reference genes in Clostridium difficile for gene expression normalization, Anaerobe, 16, 439-443 (2010).

11. Reiter, L., Kolsto, A. B., and Piehler, A. P.: Reference genes for quantitative, reverse-transcription PCR in Bacillus cereus group strains throughout the bacterial life cycle, J. Microbiol. Methods, 86, 210-217 (2011).

12. Galisa, P. S., da Silva, H. A., Macedo, A. V., Reis, V. M., Vidal, M. S., Baldani, J. I., and Simoes-Araujo, J. L.: Identification and validation of reference genes to study the gene expression in Gluconacetobacter diazotrophicus grown in different carbon sources using RT-qPCR, J. Microbiol. Methods, 91, 1-7 (2012).

13. Desroche, N., Beltramo, C., and Guzzo, J.: Determination of an internal control to apply reverse transcription quantitative PCR to study stress response in the lactic acid bacterium Oenococcus oeni, J. Microbiol. Methods, 60, 325-333 (2005).

14. Jin, C., Yao, M. F., Liu, H. F., Lee, C. F. F., and Ji, J.: Progress in the production and application of n-butanol as a biofuel, Renew. Sust. Energy Rev., 15, 4080-4106 (2011)

15. Bredwell, M. D., Srivastava, P., and Worden, R. M.: Reactor design issues for synthesis-gas fermentations, Biotechnol. Prog., 15, 834-844 (1999).

16. Heiskanen, H., Virkajarvi, I., and Viikari, L.: The effect of syngas composition on the growth and product formation of Butyribacterium methylotrophicum, Enzyme Microb. Technol., 41, 362-367 (2007).

17. Cotter, J. L., Chinn, M. S., and Grunden, A. M.: Ethanol and acetate production by Clostridium ljungdahlii and Clostridium autoethanogenum using resting cells, Bioprocess Biosyst. Eng., 32, 369-380 (2009).

18. Younesi, H., Najafpour, G., and Mohamed, A. R.: Ethanol and acetate production from synthesis gas via fermentation processes using anaerobic bacterium, Clostridium ljungdahlii, Biochem. Eng. J., 27, 110-119 (2005).
19. Köpke, M., Held, C., Hujer, S., Liesegang, H., Wiezer, A., Wollherr, A., Ehrenreich, A., Liebl, W., Gottschalk, G., and Durre, P.: Clostridium ljungdahlii represents a microbial production platform based on syngas, Proc. Natl. Acad. Sci. USA, 107, 13087-13092 (2010).

20. Köpke, M., Mihalcea, C., Liew, F., Tizard, J. H., Ali, M. S., Conolly, J. J., AlSinawi, B., and Simpson, S. D.: 2,3-butanediol production by acetogenic bacteria, an alternative route to chemical synthesis, using industrial waste gas, Appl. Environ. Microbiol., 77, 5467-5475 (2011).

21. Thomas, F., Barbeyron, T., and Michel, G.: Evaluation of reference genes for real-time quantitative PCR in the marine flavobacterium Zobellia galactanivorans, J. Microbiol. Methods, 84, 61-66 (2011).

22. Andersen, C. L., Jensen, J. L., and Orntoft, T. F.: Normalization of real-time quantitative reverse transcription-PCR data: a model-based variance estimation approach to identify genes suited for normalization, applied to bladder and colon cancer data sets, Cancer Res., 64, 5245-5250 (2004).

23. Ritz, M., Garenaux, A., Berge, M., and Federighi, M.: Determination of rpoA as the most suitable internal control to study stress response in C. jejuni by RTqPCR and application to oxidative stress, J. Microbiol. Methods, 43, 429-435 (2009).

24. Piston, F., Dorado, G., Martin, A., and Barro, F.: Cloning and characterization of a gamma-3 hordein mRNA (cDNA) from Hordeum chilense (Roem. et Schult.) Theor. Appl. Genet., 108, 1359-1365 (2004).

25. Doshi, K. M., Eudes, F., Laroche, A., and Gaudet, D.: Anthocyanin expression in marker free transgenic wheat and triticale embryos, In Vitro Cell. Dev. Biol. Plant, 43, 429-435 (2007)

26. Katayama, S., Dupuy, B., Garnier, T., and Cole, S. T.: Rapid expansion of the physical and genetic map of the chromosome of Clostridium perfringens CPN50 J. Bacteriol., 177, 5680-5685 (1995).

27. Kilian, M., Scholz, C. F., and Lomholt, H. B.: Multilocus sequence typing and phylogenetic analysis of Propionibacterium acnes, J. Clin. Microbiol., 50, 1158-1165 (2012)

28. Dheda, K., Huggett, J. F., Bustin, S. A., Johnson, M. A., Rook, G., and Zumla, A.: Validation of housekeeping genes for normalizing RNA expression in real-time PCR, Biotechniques, 37, 112-119 (2004).

29. Piehler, A. P., Grimholt, R. M., Ovstebo, R., and Berg, J. P.: Gene expression results in lipopolysaccharide-stimulated monocytes depend significantly on the choice of reference genes, BMC Immunol., 11, 21 (2010).

30. Stevenson, D. M. and Weimer, P. J.: Expression of 17 genes in Clostridium thermocellum ATCC 27405 during fermentation of cellulose or cellobiose in continuous culture, Appl. Environ. Microbiol., 71, 4672-4678 (2005).

31. Dhalluin, A., Bourgeois, I., Pestel-Caron, M., Camiade, E., Raux, G., Courtin, P., Chapot-Chartier, M. P., and Pons, J. L.: Acd, a peptidoglycan hydrolase of Clostridium difficile with $\mathrm{N}$-acetylglucosaminidase activity, Microbiology, 151, 2343-2351 (2005).

32. Deneve, C., Delomenie, C., Barc, M. C., Collignon, A., and Janoir, C.: Antibiotics involved in Clostridium difficile-associated disease increase colonization factor gene expression, J. Med. Microbiol., 57, 732-738 (2008).

33. Condon, C., Squires, C., and Squires, C. L.: Control of rRNA transcription in Escherichia coli, Microbiol. Mol. Biol. Rev., 59, 623-645 (1995).

34. Ruiz-Garbajosa, P., Bonten, M. J., Robinson, D. A., Top, J., Nallapareddy, S. R., Torres, C., Coque, T. M., Canton, R., Baquero, F., Murray, B. E., del Campo, R. and Willems, R. J.: Multilocus sequence typing scheme for Enterococcus faecalis reveals hospital-adapted genetic complexes in a background of high rates of recombination, J. Clin. Microbiol., 44, 2220-2228 (2006).

35. Wan, H., Zhao, Z., Qian, C., Sui, Y., Malik, A. A., and Chen, J.: Selection of appropriate reference genes for gene expression studies by quantitative realtime polymerase chain reaction in cucumber, Anal. Biochem., 399, 257-261 (2010).

36. Bin, W. S., Wei, L. K., Ping, D. W., Li, Z., Wei, G., Bing, L. J., Gui, P. B. Jian, W. H., and Feng, C. J.: Evaluation of appropriate reference genes for gene expression studies in pepper by quantitative real-time PCR, Mol. Breed., 30, 1393-1400 (2012) 